\title{
Catalyst-free nitro-coupling synthesis of azo-linked conjugated microporous polymers with superior aqueous energy storage capability
}

\author{
Dianlei Fang, Xinlei Wang, Shijie Zhang, Lingyan Sheng, Jian Tang, Jie Zhou ${ }^{*}$ and Weihua Tang*
}

\begin{abstract}
Developing methods to construct conjugated microporous polymers (CMPs) with desirable structures is a crucial undertaking. However, obtaining azo-CMPs with extended azaacene cores is immensely challenging. Herein, we disclose the facile synthesis of diquinoxalino[2,3-a:2',3'-c]phenazine-core CMP (3Qn-CMP; core diameter, $\sim 1.6 \mathrm{~nm})$ via the one-step hydrothermal homocoupling of nitro monomers in a mixture solution of $\mathrm{N}, \mathrm{N}$-dimethylformamide (or $\mathrm{N}, \mathrm{N}$-dimethylacetamide) and water $(1: 3, v / v)$. A $3 \mathrm{Qn}-\mathrm{CMP} / \mathrm{rGO}$ hybrid is readily prepared from CMP spheres (diameter, 0.5-1.5 $\mu \mathrm{m}$ ) anchored on reduced graphene oxide (rGO) nanosheets ( 3 layers) by synergizing the one-pot synthesis of rGO for intercalation and self-assembly. The highly porous hybrid features dual redox active sites with azo and imine linkages and efficient charge conduction. $3 \mathrm{Qn}-\mathrm{CMP}$ and $3 \mathrm{Qn}$ $\mathrm{CMP} / \mathrm{rGO}$ exhibit outstanding energy storage capacity, current density tolerance (1-50 $\left.\mathrm{A} \mathrm{g}^{-1}\right)$, and long-term cycling stability in aqueous acidic electrolytes. $3 \mathrm{Qn}-\mathrm{CMP}$ and $3 \mathrm{Qn}$ $\mathrm{CMP} / \mathrm{rGO}$ also produce high specific capacitances of 615.4 and $847.8 \mathrm{~F} \mathrm{~g}^{-1}$, respectively, at $1 \mathrm{~A} \mathrm{~g}^{-1}$. Approximately $99.1 \%$ of this capacitance can be retained over 50,000 continuous charge/discharge cycles at $50 \mathrm{~A} \mathrm{~g}^{-1}$. To the best of our knowledge, our hybrid outperforms most organic molecules or CMP-/rGO-based composites reported in the literature in terms of capacitance and cycling durability. This work provides a general strategy to access a new library of CMPs and hybrids with multilevel elaborate architectures tailored for target applications, such as electrochemical energy storage and catalysis.
\end{abstract}

Keywords: conjugated microporous polymers, nitro-coupling, azo, hydrothermal reaction, energy storage

\section{INTRODUCTION}

The strong need for electrochemical energy storage systems has invigorated research on organic electrode materials, the utilization of which is regarded as an alternative approach to address cost and environmental issues caused by inorganic electrodes, such as conventional transition metal oxides in rechargeable batteries. The structural diversity of organic electrode materials allows their functional design through various chemical synthesis routes [1-3]. However, most low-molecular weight electroactive compounds suffer from poor electronic conductivity and serious electrolyte dissolution, leading to inferior rate capacities and unacceptable cycling performance. These issues have inspired molecular engineering investigations focused on conjugation extension and polymerization. Compared with small molecules, conjugated polymers are characterized by extended skeletons that could promote efficient charge delocalization and electron transport and simultaneously maintain the integrity of the molecule in various electrolytes.

Conjugated microporous polymers (CMPs), a unique type of polymers with tunable porous morphology, have emerged as promising organic electrode candidates. CMPs are usually synthesized through the covalent linkage of $\pi$-conjugated functional units to form polymeric networks with considerable penetrable porosity, which could remarkably increase ion transport. The synthesis routes of these materials include Suzuki, Yamamoto, Buchwald-Hartwig coupling and chemical oxidative polymerization, which enable CMPs to exhibit low solubility, high structural stability, rapid electron/ion diffusion, and fast reaction kinetics [4-7].

A large number of CMPs have been explored as electrodes for electrochemical energy storage [8-10]. For example, a series of polyaminoanthraquinone networks have been successfully synthesized through Buchwald-Hartwig coupling between 2,6-diaminoanthraquinone and aryl bromides. These CMPs demonstrated average surface areas of up to $600 \mathrm{~m}^{2} \mathrm{~g}^{-1}$ [11], a specific capacitance $\left(C_{\mathrm{s}}\right)$ of $576 \mathrm{~F} \mathrm{~g}^{-1}$ in $0.5 \mathrm{~mol} \mathrm{~L}^{-1}$ aqueous $\mathrm{H}_{2} \mathrm{SO}_{4}$, and nearly $100 \%$ Coulombic efficiency over 6000 cycles at a current density of $2 \mathrm{~A} \mathrm{~g}^{-1}$. Carbon-based materials are frequently employed to prepare hybrid composites featuring enhanced charge transport. Polytriphenylamine-based networks (PTPA) have been reported to be successfully formed on carbon nanotube fibers. As-synthesized PTPA grafted on carbon nanotube fibers show high areal $C_{\mathrm{s}}\left(671.9 \mathrm{mF} \mathrm{cm}^{-2}\right.$ at $\left.1 \mathrm{~mA} \mathrm{~cm}^{-2}\right)$ [12]. The high redox activity and reaction reversibility of organic functionalities endow CMPs with superior electrochemical properties. Thus, carbonyl $(\mathrm{C}=\mathrm{O})$, imine $(\mathrm{C}=\mathrm{N})$, and triphenylamine groups have been incorporated into polymers to function as electrochemical hosts for various cations (e.g., $\mathrm{Li}^{+}, \mathrm{Na}^{+}, \mathrm{Mg}^{2+}$, $\mathrm{H}^{+}$) [13-15]. Building blocks such as quinones [16-19], phenazine $[20,21]$, and triphenylamine $[5,22]$ are usually employed as conjugated backbones. Furthermore, new redox functionalities are highly demanding to enrich the family of electrochemical CMPs. The azo group was recently discovered to be an electrochemical active site that could reversibly bond with $\mathrm{Na}^{+}$or $\mathrm{Li}^{+}$ $[23,24]$. Azobenzene derivatives have also been explored as active materials for redox flow batteries [25,26]. All redox batteries exhibited good potential for development into highcapacity and long-life energy storage systems. 
Inspired by the emerging potential applications of azo groups in energy storage, researchers developed azo-linked covalent organic frameworks (COFs) with azo-containing conjugated molecules following the conventional Schiff condensation route (Fig. 1a) [27-29]. The COFs are functionalized with either amino or aldehyde moieties for knot-joining. For instance, azo and carbonyl-modified COFs (Tp-Azo-COF) featuring dual active sites were easily synthesized from $4,4^{\prime}$-azodianiline and 1,3,5-triformylphloroglucinol [30] and delivered a capacity of $305.97 \mathrm{~mA} \mathrm{~h} \mathrm{~g}^{-1}$ at $1 \mathrm{~A} \mathrm{~g}^{-1}$ over 3000 cycles when assembled as an anode in a lithium-ion battery. However, the variety of azoCOFs is largely limited by the accessibility of azo molecules. Azo-linked CMPs could be prepared via two-step procedures from nitro monomers (Fig. 1b), including zinc-induced reductive homocoupling [31], copper(I)-catalyzed amino coupling [32], and electrophilic substitution of diazonium salts [33]. The as-obtained CMPs are amorphous in nature and mostly composed of particles measuring hundreds of nanometers in size. Thus, azo-decorated CMP nanosheets are promising candidate electrodes, but their synthesis remains a significant challenge.

In this work, we demonstrate, for the first time, the facile hydrothermal synthesis of redox azo-CMPs through the direct homocoupling of 2,8,14,-trinitro-diquinoxalino[2,3-a:2',3'-c]phenazine (3Qn). The in-situ growth of azo-CMPs on a conductive template (i.e., reduced graphene oxide, rGO) and simultaneous reduction of GO nanosheets were then explored. The as-designed $3 \mathrm{Qn}-\mathrm{CMP} / \mathrm{rGO}$ hybrid features dual redoxactive sites of $\mathrm{C}=\mathrm{N}$ and $\mathrm{N}=\mathrm{N}$ in triple fused-azaacene to promote charge storage. The hybrid CMPs produced an outstanding $C_{s}$ of $847.8 \mathrm{~F} \mathrm{~g}^{-1}$ (at $1 \mathrm{~A} \mathrm{~g}^{-1}$ ) in the $1 \mathrm{~mol} \mathrm{~L}^{-1} \mathrm{H}_{2} \mathrm{SO}_{4}$ electrolyte; such a $C_{s}$ is much higher than those of pure $3 \mathrm{Qn}-\mathrm{CMP}\left(615 \mathrm{~F} \mathrm{~g}^{-1}\right)$ and rGO $\left(125 \mathrm{~F} \mathrm{~g}^{-1}\right)$. Furthermore, ultra-high cycling stability (99.1\% capacitance retention) was achieved after 50,000 charge/ discharge cycles even at a current density of as high as $50 \mathrm{~A} \mathrm{~g}^{-1}$. Given its superior energy storage capability and cycling stability, the $3 \mathrm{Qn}-\mathrm{CMP} / \mathrm{rGO}$ electrode, to the best of our knowledge, outperforms most other redox CMP- or rGO-based composite electrodes for supercapacitors reported in the literature. Our work demonstrates a novel route for the synthesis of redox azoCMPs via the homocoupling of nitro azaacenes for use as organic electrodes with high capacitance, rate capacity, and cycling stability.

\section{EXPERIMENTAL SECTION}

\section{Synthesis of 3Qn-CMP}

A solution of $3 \mathrm{Qn}(80 \mathrm{mg})$ in $10 \mathrm{~mL}$ of $N, N$-dimethylformamide (DMF) was added with $30 \mathrm{~mL}$ of deionized water, sonicated for $30 \mathrm{~min}$, and stirred for $2 \mathrm{~h}$ at room temperature. The brown mixture was then transferred to a 50-mL Teflon-lined stainlesssteel autoclave for hydrothermal treatment at $180^{\circ} \mathrm{C}$ for $12 \mathrm{~h}$. The mixture was cooled, filtered, and washed several times with ethanol and deionized water. The collected product was soaked in tetrahydrofuran, acetone, and petroleum ether in sequence for $30 \mathrm{~min}$ each time. Freeze-drying afforded spheres as a black solid ( $\sim 73 \mathrm{mg}$, yield: $\sim 91 \%)$. Solid-state ${ }^{13} \mathrm{C}$ nuclear magnetic resonance spectroscopy of azo-CMPs confirmed the presence of characteristic aromatic carbons of diquinoxalino[2,3-a:2',3'-c]phenazine in the range of $100-150$ ppm (Fig. S1).

\section{Synthesis of rGO}

A suspension of GO (40 mg) in $40 \mathrm{~mL}$ of deionized water was sonicated for $30 \mathrm{~min}$ and then transferred to a $50-\mathrm{mL}$ Teflonlined stainless-steel autoclave. The hydrothermal reaction was conducted at $180^{\circ} \mathrm{C}$ for $12 \mathrm{~h}$. Thereafter, the rGO hydrogels were cooled and washed several times with ethanol and deionized water. rGO was obtained after freeze-drying $(\sim 30 \mathrm{mg}$, yield: $\sim 75 \%)$.

\section{Synthesis of $3 Q \mathrm{n}-\mathrm{CMP} / \mathrm{rGO}$}

GO $(40 \mathrm{mg})$ was added to deionized water $(30 \mathrm{~mL})$, and $3 \mathrm{Qn}$ $(80 \mathrm{mg})$ was added to DMF $(10 \mathrm{~mL})$. The two solutions were

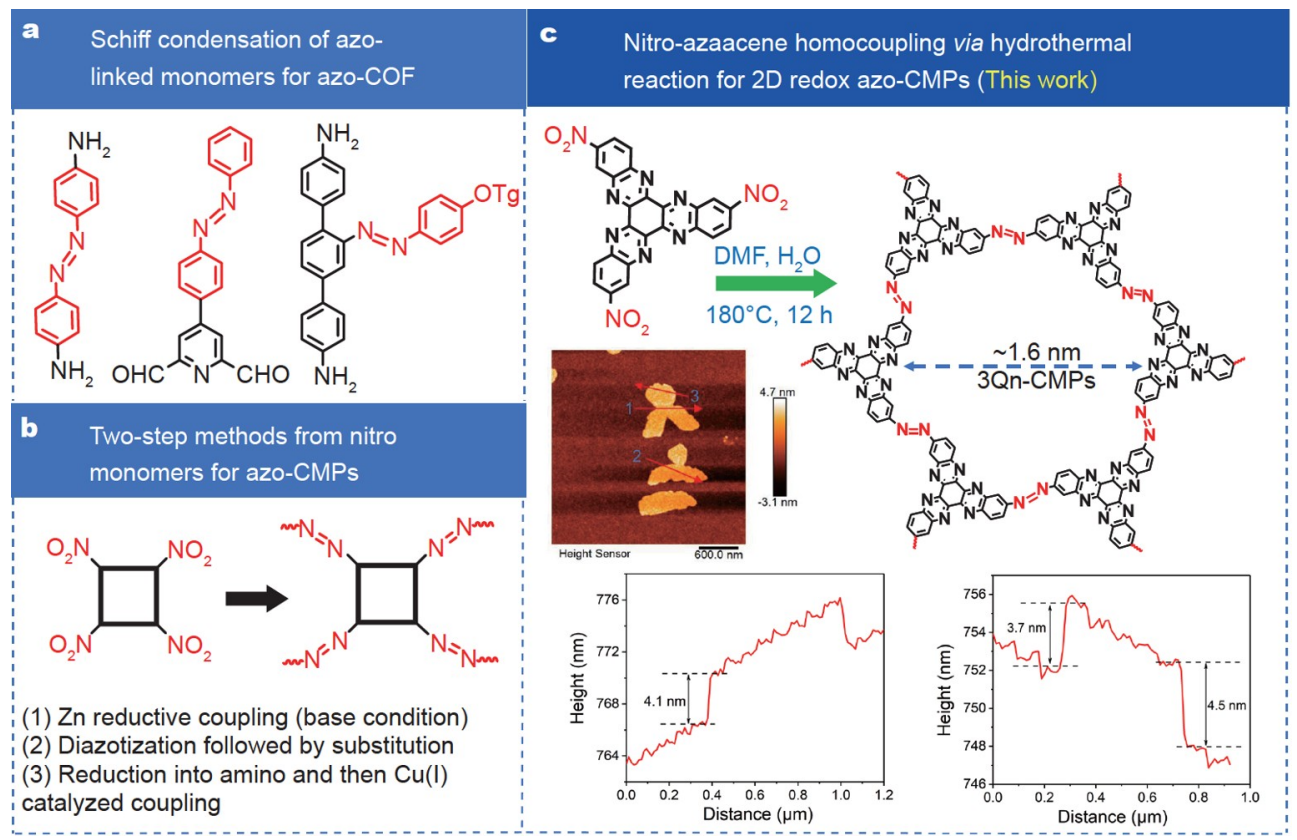

Figure 1 (a) Condensation of imine-linked azo-containing COFs. (b) Two-step synthetic strategies for obtaining azo-linked CMPs. (c) Direct synthesis of azo-azaacene CMPs from the homocoupling of nitro-azaacene. 
mixed and vigorously stirred for $15 \mathrm{~min}$. The mixture was then sonicated for $30 \mathrm{~min}$ to enrich and assemble the $3 \mathrm{Qn}$ molecules on the GO layers. The mixture was transferred to a $50-\mathrm{mL}$ Teflon-lined stainless-steel autoclave and heated at $180^{\circ} \mathrm{C}$ for $12 \mathrm{~h}$. The autoclave was cooled to room temperature to afford the $3 \mathrm{Qn}-\mathrm{CMP} / \mathrm{rGO}$ hydrogels, which were thoroughly washed several times with ethanol and deionized water. The product was then soaked in tetrahydrofuran, acetone, and petroleum ether for $30 \mathrm{~min}$ to remove impurities. Freeze-drying afforded the target 3Qn-CMP/rGO hybrid as a black solid ( 114 mg, yield: $\sim 95 \%)$.

\section{RESULTS AND DISCUSSION}

\section{Materials characterization}

The star-shaped redox $3 \mathrm{Qn}$ was readily synthesized by the condensation of hexaketocyclohexane with 4-nitro-o-phenylenediamine in $\sim 72 \%$ yield; the synthetic procedure of the monomer is depicted in Scheme S1 and Figs S2-S4 [34]. Redox azo-CMPs were further synthesized via the catalyst-free hydrothermal nitro-coupling of $3 \mathrm{Qn}$ at $180^{\circ} \mathrm{C}$ for $12 \mathrm{~h}$ (Fig. 1c). Direct azo conversion critically relies on the reaction solvents. The mixture solution of DMF:water $(1: 3, v / v)$ or $N, N$-dimethylacetamide (DMAc):water $(1: 3, v / v)$ could successfully afford the title $3 \mathrm{Qn}-\mathrm{CMP}$ as black polymers in high yields (>90\%). These polymers are insoluble in common organic solvents and water and, thus, could easily be purified by solvent washing or Soxhlet extraction. The conversion of azo-CMPs was unsuccessful when a single solvent, such as water, DMF, DMAc, or dimethyl sulfoxide (DMSO), was used. 3Qn-CMPs could be fabricated into three-dimensional (3D) architectures for practical applications via the in-situ growth of 3Qn-CMPs on GO sheets. This strategy is built on the principle of "killing two birds with one stone", i.e., promoting the assembly of azaacene with oxygen-rich GO nanosheets and improving the electric conductivity of the hybrid with the formed rGO. The presence of GO does not affect the formation of CMPs but promotes the conversion of monomers via its oxygen-containing functionalities instead.

The crystal structure of 3Qn-CMP was evaluated via structural simulation and powder X-ray diffractometry (PXRD). By minimizing the geometrical energy implemented in the Materials Studio software package, we acquired unit cell parameters of $a=$ $b=28.078 \AA, c=3.321 \AA, \alpha=\beta=90^{\circ}$, and $\gamma=120^{\circ}$ (Fig. 2a). Full profile pattern matching (Pawley) refinements were applied to the experimental PXRD patterns (Fig. 2b), and the refinement results matched the observed ones well with good agreement factors $\left(R_{\mathrm{p}}=3.91 \% ; \omega R_{\mathrm{p}}=5.00 \%\right) .3 \mathrm{Qn}$-CMP showed typical (110) and (001) peaks at $6.0^{\circ}$ and $26.9^{\circ}$, respectively. The 3Qn$\mathrm{CMP} / \mathrm{rGO}$ remains the strong peak for the (001) plane, together with broadened and shifted peak of the (110) plane due to the rGO substrate (Fig. S5a). The characteristic peak of rGO at $26^{\circ}$, which reflects the (002) plane, disappeared in the spectrum of the hybrid. The successful hydrothermal reduction of GO into rGO is confirmed by the pronounced shift of the (002) peak, which is due to the loss of oxygen-containing groups and restacking of graphene sheets [35]. The sharp peak at $10.9^{\circ}$, which reflects the (002) plane of typical carbonaceous materials, is observed in the spectrum of pristine GO (Fig. S5b). This phenomenon is consistent with the imine-linked $\mathrm{CMP} / \mathrm{rGO}$ case [36]. The crystalline peaks of the $3 \mathrm{Qn}$ monomer disappeared in pure $3 \mathrm{Qn}-\mathrm{CMP}$ and $3 \mathrm{Qn}-\mathrm{CMP} / \mathrm{rGO}$, thus implying the complete conversion of CMPs.

The porosity of the as-prepared hybrids was investigated using nitrogen adsorption/desorption isotherms. A pore diameter distribution of $1.6 \mathrm{~nm}$ was calculated for $3 \mathrm{Qn}-\mathrm{CMP}$, consistent with the simulated results (Fig. S6). The specific surface area of $3 \mathrm{Qn}-\mathrm{CMP} / \mathrm{rGO}$ was measured to be $44.3 \mathrm{~m}^{2} \mathrm{~g}^{-1}$ (Fig. S7), which is larger than those of $\mathrm{rGO}\left(31.9 \mathrm{~m}^{2} \mathrm{~g}^{-1}\right)$ and $3 \mathrm{Qn}-\mathrm{CMP}$

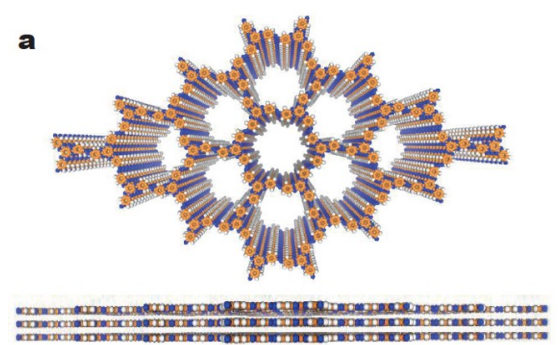

$a=b=28.078 \AA, c=3.321 \AA$

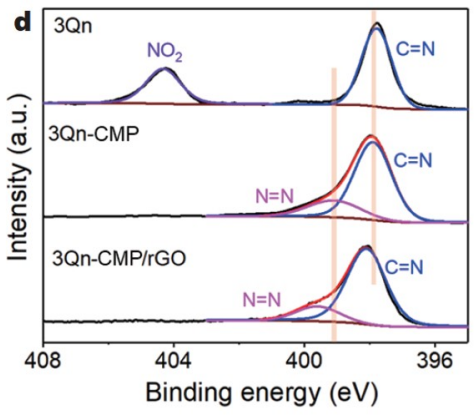

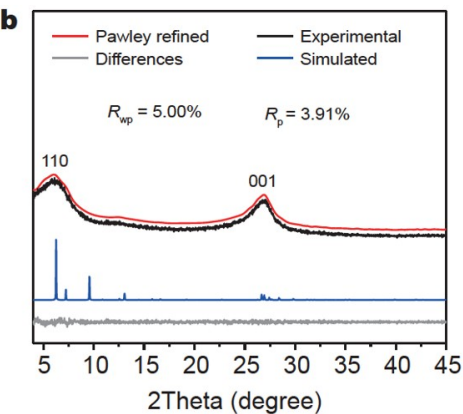

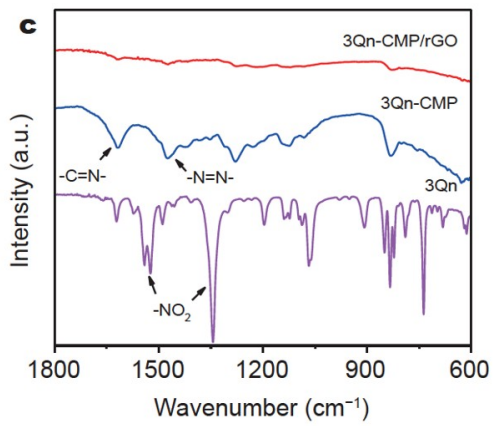

e
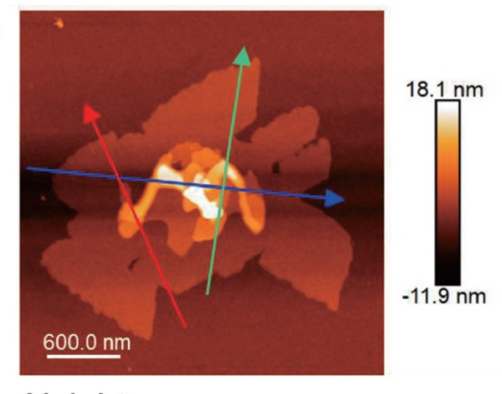

Height sensor

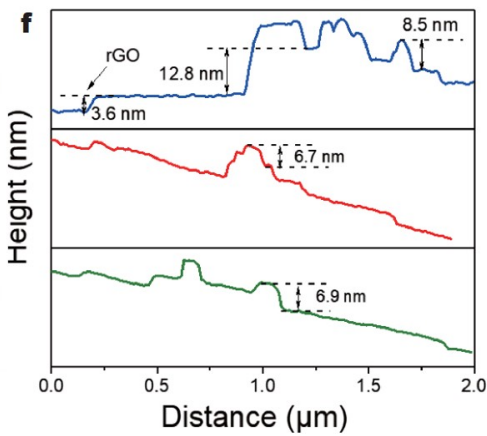

Figure 2 (a) Schematic representation of 3Qn-CMP. (b) PXRD patterns of the experimental and simulated eclipsed patterns of 3Qn-CMP. (c) Magnified FTIR spectra. (d) N 1s spectra of 3Qn, 3Qn-CMP, and 3Qn-CMP/rGO. (e) AFM image and (f) the height profiles of 3Qn-CMP/rGO. 
$\left(22.6 \mathrm{~m}^{2} \mathrm{~g}^{-1}\right)$. The relatively low Brunauer-Emmett-Teller (BET) surface area of $3 \mathrm{Qn}-\mathrm{CMP}$ and $3 \mathrm{Qn}-\mathrm{CMP} / \mathrm{rGO}$ compared with those of previously reported CMPs could be attributed to the low crystallinity of our sample. Earlier studies indicated that low crystallinity could influence the accessibility of pathways for nitrogen gas, resulting in low BET values [37].

The evolution of nitro groups in response to hydrothermal treatment was monitored using Fourier-transform infrared spectroscopy (FT-IR) and X-ray photoelectron spectroscopy (XPS). As depicted in Fig. S8, GO exhibited a series of characteristic FT-IR peaks at 1729, 1629, 1233, and $1044 \mathrm{~cm}^{-1}$, which correspond to the bond vibrations of $\mathrm{C}=\mathrm{O}, \mathrm{C}=\mathrm{C}, \mathrm{C}-\mathrm{O}-\mathrm{C}$, and $\mathrm{C}-\mathrm{O}$, respectively [35]. As expected, rGO exhibited dramatically decreased peak intensities for $\mathrm{C}=\mathrm{O}$ and $\mathrm{C}-\mathrm{O}$ bond vibrations, thus confirming the efficient hydrothermal reduction of GO. Two strong FT-IR peaks at 1525 and $1343 \mathrm{~cm}^{-1}$ were observed in the FT-IR spectrum of $3 \mathrm{Qn}$, and these peaks were respectively attributed to the asymmetric and symmetric stretching vibrations of the $-\mathrm{NO}_{2}$ group (Fig. 2c) [38]. These peaks disappeared and a new peak representing the vibrations of the $\mathrm{N}=\mathrm{N}$ group $\left(1475 \mathrm{~cm}^{-1}\right)$ emerged in the spectra of $3 \mathrm{Qn}$ CMP and 3Qn-CMP/rGO [28]. The imine peak at $\sim 1620 \mathrm{~cm}^{-1}$ was well preserved. The high-resolution XPS spectrum of $\mathrm{N} 1 \mathrm{~s}$ of $3 \mathrm{Qn}$ could be deconvoluted into nitro $(404.3 \mathrm{eV})$ and imine $(397.8 \mathrm{eV})$ groups (Fig. 2d) [24,39]. The XPS spectra of 3Qn$\mathrm{CMP}$ and $3 \mathrm{Qn}-\mathrm{CMP} / \mathrm{rGO}$ showed the complete disappearance of the peak for the $-\mathrm{NO}_{2}$ group, which indicates the successful conversion. The broad peak of $\mathrm{N} 1 \mathrm{~s}$ at $399.1 \mathrm{eV}$ could be deconvoluted into the imine group $(\mathrm{C}=\mathrm{N})$ at $397.8 \mathrm{eV}$, the azo group $(\mathrm{N}=\mathrm{N})$ at $398.7 \mathrm{eV}$, and the nitroso $(\mathrm{N}=\mathrm{O})$ group at $400.6 \mathrm{eV}$ [24,40-42]. According to the mechanism proposed for the gold-catalyzed reduction of nitro compounds, which is achieved through either the direct route via nitroso and hydroxylamine intermediates or the condensation route via azoxy and azo intermediates [43], the reduction of nitro into azo functionalities in our case could reasonably be inferred to generate the nitroso residues of $3 \mathrm{Qn}-\mathrm{CMP}$. The presence of a certain amount of nitroso groups (ca. $<10 \%)$ could be observed in the full-survey XPS spectra of $3 \mathrm{Qn}-\mathrm{CMP}$ and $3 \mathrm{Qn}-\mathrm{CMP} / \mathrm{rGO}$ (Fig. S9). The slightly higher intensity of the $\mathrm{O}$ 1s peak in the $3 \mathrm{Qn}-\mathrm{CMP} / \mathrm{rGO}$ spectrum relative to that in the $3 \mathrm{Qn}-\mathrm{CMP}$ spectrum indicates a small number of oxygen-containing groups (e.g., $\mathrm{C}=\mathrm{O}, \mathrm{C}-\mathrm{O}$, and $-\mathrm{OH}$ ) in the $\mathrm{GO}$ residues after reduction (Fig. S10). The Raman spectrum (Fig. S11) of 3Qn-CMP exhibited peaks similar to those of the D/G band of graphite on account of the extensive conjugated fused ring structure of the former [44]. 3Qn-CMP clearly has a lower $I_{\mathrm{D}} / I_{\mathrm{G}}$ ratio than $\mathrm{rGO}$, which indicates its more orderly arrangement. By comparison, $3 \mathrm{Qn}-\mathrm{CMP} / \mathrm{rGO}$ shows an intermediate $I_{\mathrm{D}} / I_{\mathrm{G}}$ ratio, thereby indirectly reflecting the successful assembly and combination of its individual components. Atomic force microscopy (AFM) revealed that pristine azo-CMP presents as $2 \mathrm{D}$ nanosheets with a thickness of 3.7-5.4 nm (Fig. S12). The azo-CMP/rGO hybrid showed the presence of aggregated CMP on the rGO nanosheets (Fig. 2e). Considering the thicknesses of GO $(0.8-1.2 \mathrm{~nm}$, according to the product label) and rGO (2.1-3.6 nm) (Fig. S13), the azo-CMP/rGO hybrid featured 6.9-12.8-nm-thick azo-CMP lamina accommodated on approximately three-layer stacked rGO nanosheets. These findings confirm that our hydrothermal strategy could simultaneously generate azo-CMP and rGO with high efficiency.
To achieve the desired intercalation architecture, we optimized the synthesis procedure for both azo-CMPs and the azo$\mathrm{CMP} / \mathrm{rGO}$ hybrid by using ultrasonication. The effects of $\pi-\pi$ stacking allow the planar $3 \mathrm{Qn}$ molecules to self-assemble onto the GO nanosheets via intramolecular $\pi-\pi$ interactions, hydrogen bonding, and hydrophobic effects as driving forces (Fig. 3a) $[45,46]$. We evaluated the effect of the graphene substrate on the $3 \mathrm{Qn}$ conversion by investigating the ultraviolet-visible (UV-vis) absorption spectra of the solutions after the preparation of $3 \mathrm{Qn}$ $\mathrm{CMP}$ and 3Qn-CMP/rGO from DMF/ $\mathrm{H}_{2} \mathrm{O}(1: 3, v / v)$ (Fig. S14). Typical peaks centered at 301 and $387 \mathrm{~nm}$ could be ascribed to the $n-\pi^{*}$ and $\pi-\pi^{*}$ transitions of $3 \mathrm{Qn}$ originating from $-\mathrm{NO}_{2}$ groups and large conjugated structures, respectively. These peaks nearly completely disappeared in the filtrates of the 3Qn-CMP/ rGO system and decreased by over $90 \%$ in those of pristine $3 \mathrm{Qn}$ $\mathrm{CMP}$, thereby indicating the highly effective nitro-coupling of 3Qn with GO. High GO-aided conversion yields were also revealed by changes in the peak intensity and solution color shown in the inset images in Fig. S14. The intercalation of 3Qn and its sequential in-situ conversion into 3Qn-CMP between rGO layers could readily afford the as-prepared $3 \mathrm{Qn}-\mathrm{CMP} / \mathrm{rGO}$ as hydrogel composites (Fig. 3a and Fig. S15).

The 3D morphology of the CMP particle-intercalating rGO nanosheets was clearly observed by scanning electron microscopy (SEM) and transmission electron microscopy (TEM). The rGO sheets featured crumpled surfaces and a porous network (Fig. 3b-d). Pristine 3Qn-CMP demonstrated a unique spherical shape with diameters of $0.5-1.5 \mu \mathrm{m}$ (Fig. $3 \mathrm{e}-\mathrm{g}$ ). Interestingly, the 3Qn-CMP/rGO hybrid showed hierarchically interconnected networks without obvious aggregates (Fig. $3 \mathrm{~h}-\mathrm{j}$ ). The 3Qn-CMP spheres were sandwiched between the wrinkled rGO sheets without aggregation, thereby demonstrating their close-knit incorporation. This unique morphology could accelerate ion insertion and transfer. The induction of conjugate macromolecular self-assembly and in-situ growth into spheres on the rGO sheets observed in this work are consistent with our previous study [46]. In the magnified SEM image shown in Fig. 3i, we found that the organic spheres are tightly adhered to the rGO sheet, which could improve the anchoring stability of the CMPs. Point-to-surface contact could enhance the vertical charge transport capability of the composite and support its application as electrodes for energy storage.

\section{Electrochemical measurements}

The as-designed 3Qn-CMP featuring dual redox active sites (i.e., azo and imine groups) was first investigated via cyclic voltammetry (CV) at $5 \mathrm{mV} \mathrm{s}^{-1}$ in aqueous $1 \mathrm{~mol} \mathrm{~L}^{-1} \mathrm{H}_{2} \mathrm{SO}_{4}$. A direct comparison with $\mathrm{rGO}$ and $3 \mathrm{Qn}-\mathrm{CMP} / \mathrm{rGO}$ was also performed (Fig. 4a). rGO displayed typical electron double-layer capacitor behavior within the potential window of $-0.4-0.8 \mathrm{~V}$ ( $v s . \mathrm{Ag} /$ $\mathrm{AgCl}$ ) [47]. 3Qn-CMP exhibited two pairs of redox peaks at $-0.22 /-0.05 \mathrm{~V}$ and $-0.08 / 0.05 \mathrm{~V}$, respectively. Since 3Qn molecules present only one pair of redox peaks at $-0.16 / 0.03 \mathrm{~V}$, which originate from the proton-coupled reactions of $\mathrm{C}=\mathrm{N}$ bonds (Fig. S16), the contribution of azo groups to charge storage is validated. Owing to enhanced charge and ion transport, the redox reactions of imine and azo groups were more obvious, with slight shifts toward less negative potentials. The peak shift suggests the occurrence of partial charge transfer between $3 \mathrm{Qn}$ CMP and rGO, which promotes the electro-reduction of the redox reactions of organic species [48]. 3Qn-CMP/rGO exhi- 
a

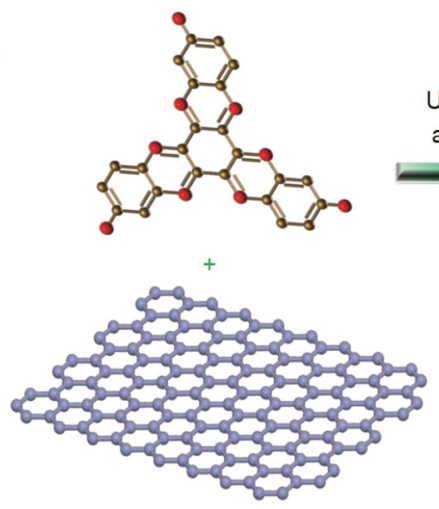

$3 Q n+G O$

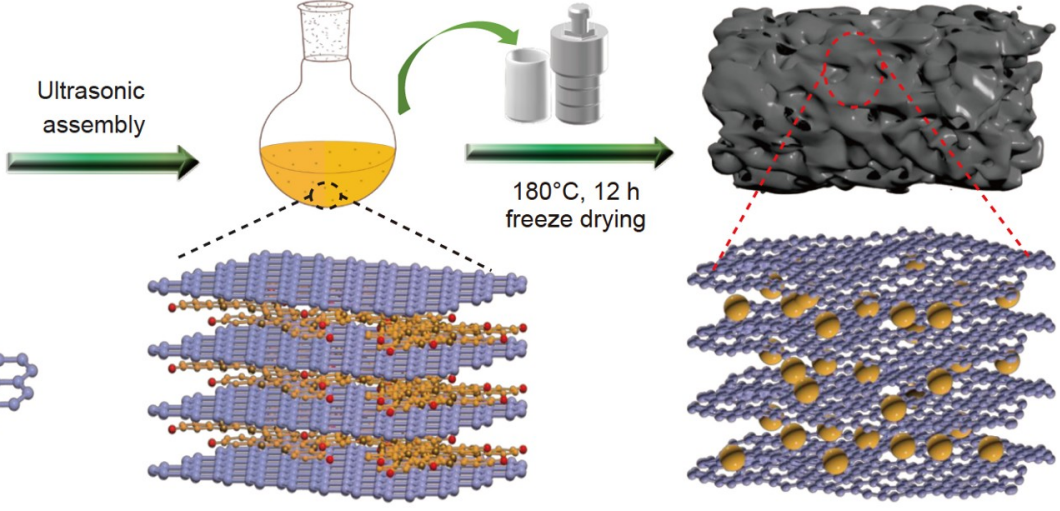

Suspension
3Qn-COF/rGO
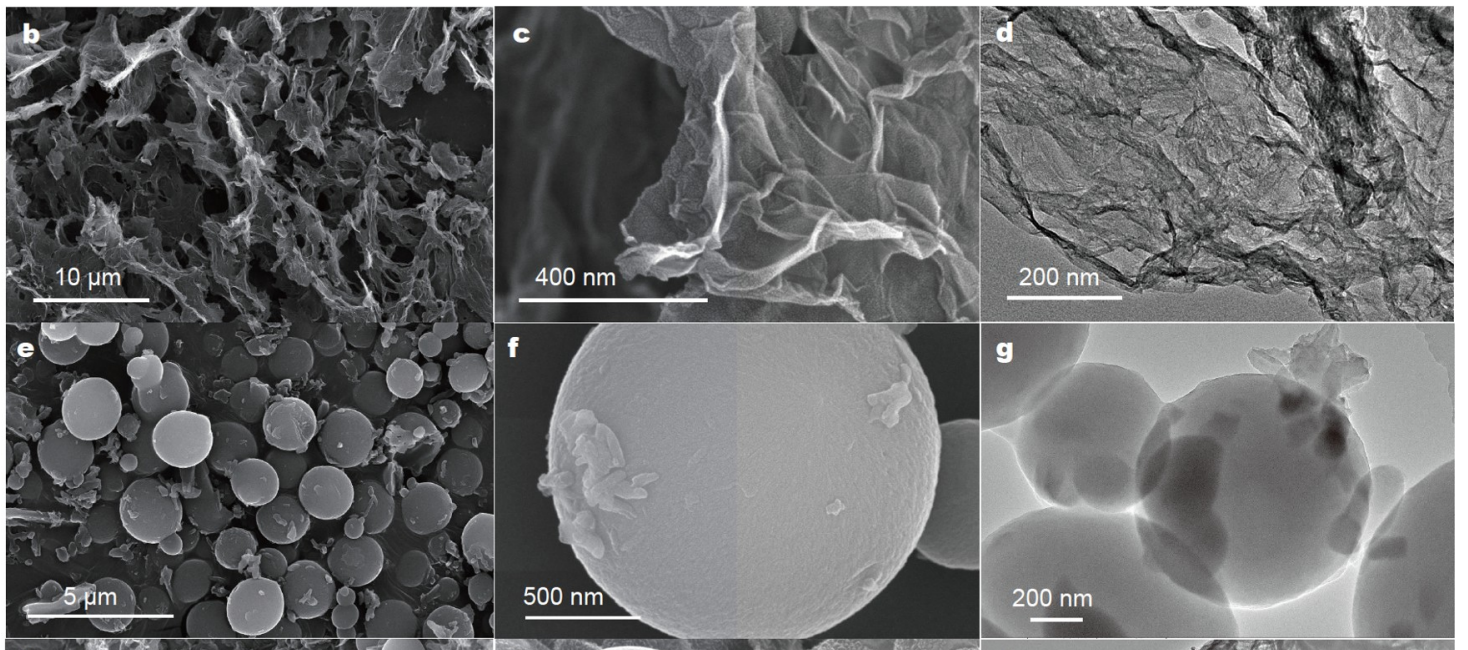

g

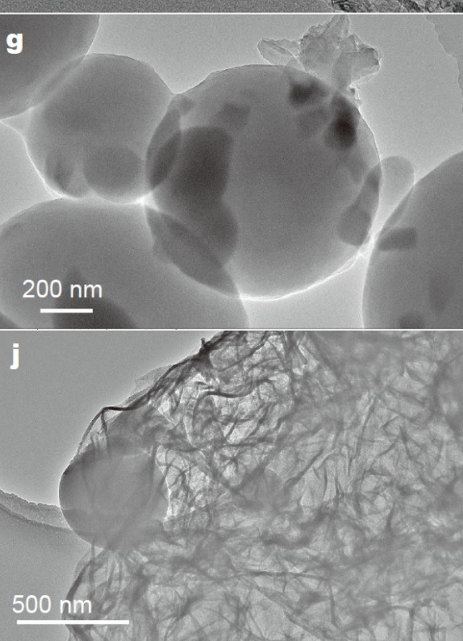

Figure 3 (a) Schematic illustration of the assembly of CMPs on rGO. (b, c) SEM and (d) TEM images of rGO. (e, f) SEM and (g) TEM images of 3Qn-CMP. (h, i) SEM and (j) TEM images of 3Qn-CMP/rGO.

bited a fairly large CV integration area, which indicates good charge storage capability.

Electrochemical impedance spectroscopy (EIS) was performed to gain insights into the enhanced electrochemical performance of $3 \mathrm{Qn}-\mathrm{CMP} / \mathrm{rGO}$ (Fig. S17). The internal resistance $\left(R_{\mathrm{s}}\right)$ and charge-transfer resistance $\left(R_{\mathrm{ct}}\right)$ determined from the fitted EIS plots are presented in Fig. $4 \mathrm{~b}, \mathrm{c}$. The $R_{\mathrm{s}}$ and $R_{\mathrm{ct}}$ of 3Qn-CMP/ rGO were smaller than those of $3 \mathrm{Qn}-\mathrm{CMP}$, which reveals improvements in electron transfer at the electrode-electrolyte interface of the hybrid electrode. Furthermore, plotting of the real part of the collected impedance $\left(Z^{\prime}\right)$ as a function of the reciprocal square root of the sampling frequency (Fig. 4d) revealed a smaller slope for $3 \mathrm{Qn}-\mathrm{CMP} / \mathrm{rGO}(0.03)$ compared with those for rGO (1.7) and 3Qn-CMP (0.17). Because the slope of this curve is correlated with ion diffusion impedance [49], $3 \mathrm{Qn}-\mathrm{CMP} / \mathrm{rGO}$ clearly shows the fastest ion transfer among the samples, which is beneficial for the redox reactions of dual active sites.

The charge/discharge behaviors of as-prepared electrodes were further evaluated by gradually increasing the current density from 1 to $50 \mathrm{~A} \mathrm{~g}^{-1}$. The galvanostatic charge-discharge (GCD) profiles of $3 \mathrm{Qn}-\mathrm{CMP} / \mathrm{rGO}$ exhibited the largest areas at different current densities, which agrees well with the $\mathrm{CV}$ measurements (Figs S18-S20). The variation of $C_{\mathrm{s}}$ with the current density is plotted in Fig. 4e. 3Qn-CMP/rGO exhibited excellent $C_{\text {s }}$ and rate capability over a wide current density range $\left(1-50 \mathrm{~A} \mathrm{~g}^{-1}\right)$. According to the discharge curves, $3 \mathrm{Qn}-\mathrm{CMP}$ and $\mathrm{rGO}$ were calculated to offer a gravimetric $C_{\mathrm{s}}$ of 615.4 and $125 \mathrm{~F} \mathrm{~g}^{-1}$ at $1 \mathrm{~A} \mathrm{~g}^{-1}$, respectively. Owing to its synergetic charge storage capability, 3Qn-CMP/rGO could deliver a rather high $C_{\mathrm{s}}$ of $847.8 \mathrm{~F} \mathrm{~g}^{-1}$, which is 1.4- and 5.8-fold higher than those of $3 \mathrm{Qn}$ CMP and $\mathrm{rGO}$, respectively. The rapid deterioration of the rate performance of pure $3 \mathrm{Qn}-\mathrm{CMP}$ may be attributed to its poor electrical conductivity $\left(4.4 \%\right.$ retention at $\left.50 \mathrm{~A} \mathrm{~g}^{-1}\right)$. Fortunately, 

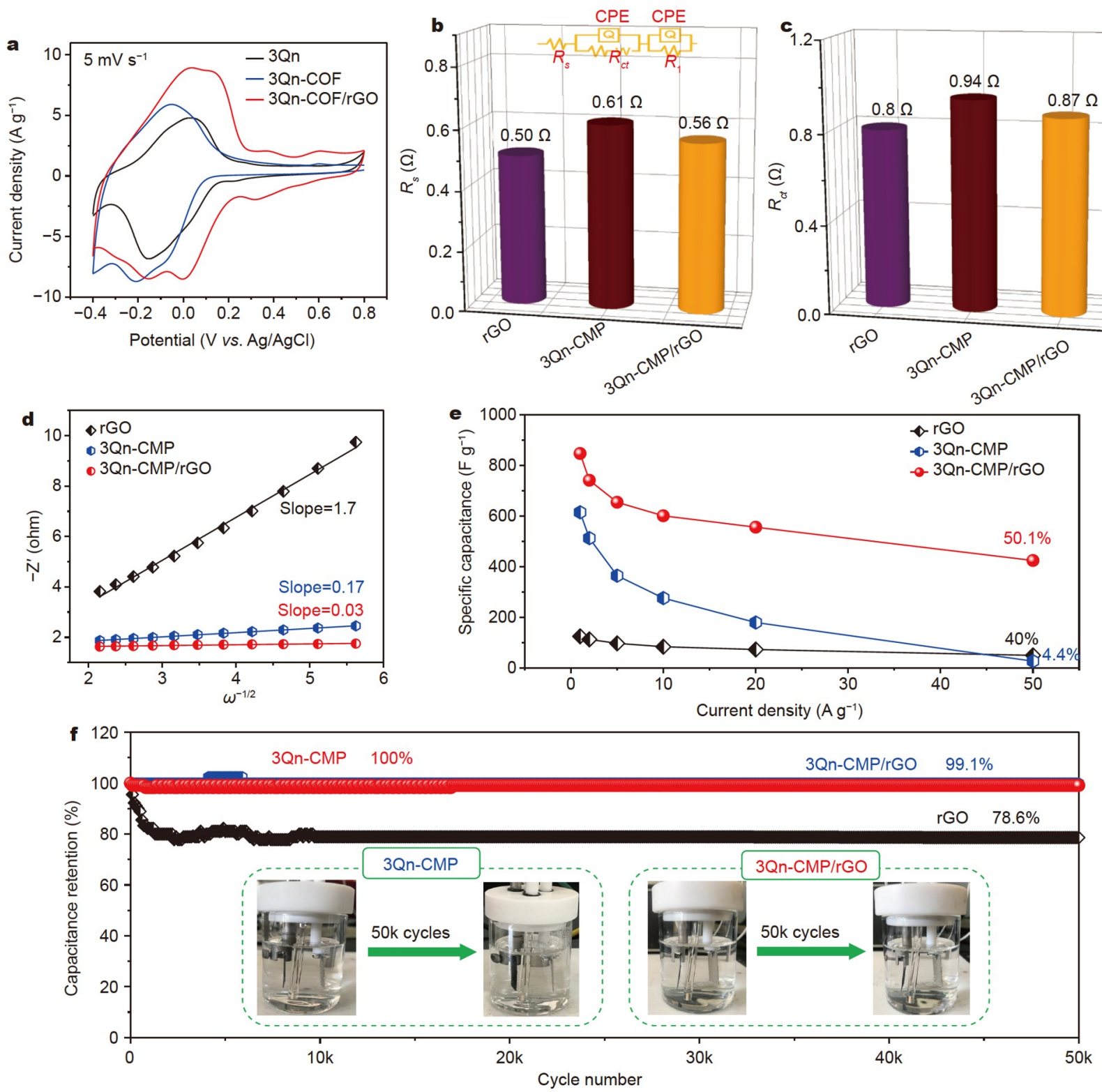

Figure 4 Electrochemical performance of rGO, 3Qn-CMP, and 3Qn-CMP/rGO. (a) CV curves obtained at a scan rate of $5 \mathrm{mV} \mathrm{s}{ }^{-1}$. (b, c) $R_{\mathrm{s}}$ and $R_{\mathrm{ct}}$ calculated from the fitted EIS plots. (d) Variations and fittings between $Z_{\mathrm{re}}$ and the reciprocal square root of the angular frequency in the low-frequency region. (e) Gravimetric specific capacitances at different current densities. (f) Cycling performance at $50 \mathrm{~A} \mathrm{~g}^{-1}$. The insets show the electrolytes of the 3Qn-CMP and $3 \mathrm{Qn}-\mathrm{CMP} / \mathrm{rGO}$ electrodes before and after 50,000 charge/discharge cycles.

the electron transfer ability of 3Qn-CMP can be compensated by the integration of $\mathrm{rGO}$ as a conductive substrate. Up to $50.1 \%$ of the $C_{s}$ of $3 \mathrm{Qn}-\mathrm{CMP} / \mathrm{rGO}\left(425 \mathrm{~F} \mathrm{~g}^{-1}\right)$ could be retained at a current density of as high as $50 \mathrm{~A} \mathrm{~g}^{-1}$. Given its outstanding rate capability over a wide current density window of $1-50 \mathrm{~A} \mathrm{~g}^{-1}$, 3Qn-CMP/rGO outperforms most other efficient organic materials, including CMP- and rGO-based electrodes (Tables S1 and S2).

Reversible redox functionalities and robust nanostructures endowed 3Qn-CMP/rGO with good electrochemical durability. The cycling performance of the $3 \mathrm{Qn}-\mathrm{CMP} / \mathrm{rGO}$ electrode was compared with those of $\mathrm{rGO}$ and 3Qn-CMP via long-term GCD tests (50,000 cycles) at $50 \mathrm{~A} \mathrm{~g}^{-1}$. As depicted in Fig. 4f, 3Qn$\mathrm{CMP} / \mathrm{rGO}$ and $3 \mathrm{Qn}-\mathrm{CMP}$ similarly showed ultra-high cycling stability with capacitance retentions of $99.1 \%$ and $100 \%$, respectively; these values are much higher than that of rGO (78.6\%). The inset in the Fig. 4f shows the photographs of the electrochemical cells, which reveal no 3Qn-CMP dissolution into the electrolyte during long-term cycling operation. By contrast, $3 \mathrm{Qn}$ molecules tended to fall off from the electrode and completely dissolve into the electrolyte during the first charge and discharge cycle (Fig. S21). This behavior could be explained by the presence of highly reactive unpaired electrons in $3 \mathrm{Qn}$, which may promote the side reactions of organic radicals and cause rapid dissolution during the GCD process. 9,10-Anthraquinone radicals have been reported to be very unstable in aqueous electrolytes [50]. Interestingly, such radicals could be efficiently stabilized in extended $\pi$-conjugated CMP backbones and polycyclic hydrocarbons [19] without sacrificing their electrochemical reactivity. Expanded $\pi-\pi$ and $p-\pi$ conjugation 
interactions are beneficial to delocalizing the energy density of unpaired electrons. Intermolecular electron self-exchange behaviors can also be restricted by steric effects [15]. In the present case, the large azo-linked azacene molecule could effectively induce electron delocalization over the extended CMP conjugated skeleton, thereby stabilizing the electroactive intermediates of long-term GCD cycling. Such behavior was schematically illustrated and compared with the theoretical calculations (Fig. S22). The outstanding electrochemical property of $3 \mathrm{Qn}-\mathrm{CMP} / \mathrm{rGO}$ could be attributed, at least in part, to the harmonious synergy between azo-CMP and rGO [51-53]. rGO sheets provide the $3 \mathrm{Qn}$ monomers with steady polymerization substrates, which not only stabilize $\pi$-conjugated fused ring molecular planes but also enhance the electronic conductivity. 3Qn-CMP, in turn, increases the charge storage ability of the hybrid electrode via its abundant redox sites. These substrates could also prevent rGO sheets from aggregating and promote ionic penetration, which further decreases the interfacial ion diffusion resistance of $3 \mathrm{Q} n-\mathrm{CMP} / \mathrm{rGO}$. These factors endow 3Qn-CMP/rGO with high capacitance, rate capability, and cycling stability.

Given its ideal nanostructure and multifunctional redox sites, the $3 \mathrm{Qn}-\mathrm{CMP} / \mathrm{rGO}$ electrode demonstrated outstanding electrochemical energy storage performance. Comparisons of the $C_{s}$, rate capability, and cycle stability between our electrodes and representative organic molecules/rGO, CMPs, and COFs published in the literature were performed (Tables S1-S3; all references are listed in the Supplementary information). As shown in Fig. 5a, b, 3Qn-CMP/rGO exhibits much more superior capacitance compared with the reported organic electrodes over a wide range of current densities (1-50 $\mathrm{A} \mathrm{g}^{-1}$ ). For instance, anthraquinone-2-sulfonate (AQS)@rGO showed a maximum $C_{\mathrm{s}}$ of $567.1 \mathrm{~F} \mathrm{~g}^{-1}$ at $1 \mathrm{~A} \mathrm{~g}^{-1}$ and a capacitance retention of $89.1 \%$ at $10 \mathrm{~A} \mathrm{~g}^{-1}$ over 10,000 cycles [54]. $p$-Phenylenediamine-modified $\mathrm{GO} / \mathrm{rGO}$ delivered a maximum $C_{\mathrm{s}}$ of $316.54 \mathrm{~F} \mathrm{~g}^{-1}$ at $10 \mathrm{mV} \mathrm{s}^{-1}$ and a capacitance retention of $93.66 \%$ over 4000 cycles at $2 \mathrm{~A} \mathrm{~g}^{-1}$ [55]. The representative CMP synthesized via Buchwald-Hartwig coupling between 2,6-diaminoanthraquinone and 1,3,5-tris(4-bromophenyl)benzene (PAQTA) delivered a maximum $C_{\mathrm{s}}$ of $576 \mathrm{~F} \mathrm{~g}^{-1}$ at $1 \mathrm{~A} \mathrm{~g}^{-1}$ and $85 \%$ capacitance retention after 6000 cycles at $2 \mathrm{~A} \mathrm{~g}^{-1}$ [11]. The performance of our CMP is superior to that of COF-based electrodes (Table S3 and Fig. S23). TpDq-COF/rGO from in situ reaction of 1,3,5-triformylphloroglucinol (Tp) and diaminoanthraquinone $(\mathrm{Dq})$ in presence of $\mathrm{GO}$ exhibited a maximum $C_{\mathrm{s}}$ of $269 \mathrm{~F} \mathrm{~g}^{-1}$ at $0.5 \mathrm{~A} \mathrm{~g}^{-1}$ and a capacitance retention of $83 \%$ after 5000 cycles at $10 \mathrm{~A} \mathrm{~g}^{-1}$ [36], while hydroquinone-based COF $\left(\mathrm{TpPa}-(\mathrm{OH})_{2}\right.$-COF) showed a $C_{\mathrm{s}}$ of $416 \mathrm{~F} \mathrm{~g}^{-1}$ at $0.5 \mathrm{~A} \mathrm{~g}^{-1}$ and a capacitance retention of only $66 \%$ after 10,000 cycles at $0.8 \mathrm{~A} \mathrm{~g}^{-1}$ [56]. Most reported COFs can tolerate current densities of up to $10 \mathrm{~A} \mathrm{~g}^{-1}$ only. The electrochemical stability of redox molecules or CMPs is largely improved by the presence of graphene. The effects of $\pi-\pi$ stacking between conjugated molecules and carbon substrates play a critical role in stabilizing organic radicals. Therefore, over $80 \%$ capacitance retention could be realized for these cases even after 10,000 cycles (Fig. 5c). Representative CMP networks have been reported to achieve capacitance retentions in the range of $80 \%-95 \%$ over 10,000 cycles (Fig. $5 d$ ). By comparison, our $3 \mathrm{Q} n-\mathrm{CMP} / \mathrm{rGO}$ exhibited outstanding stability even after 50,000 cycles on account of its dual stabilized pathways. A $3 \mathrm{Q} n-\mathrm{CMP} / \mathrm{rGO}$ symmetric supercapacitor (SSC) was assembled, and the CV and GCD curves of this device demonstrated typical pseudocapacitance behaviors (Fig. S24a, b). In addition, the device exhibited a capacitance of $187 \mathrm{~F} \mathrm{~g}^{-1}$ at $1 \mathrm{~A} \mathrm{~g} \mathrm{~g}^{-1}$ and retained $61 \mathrm{~F} \mathrm{~g}^{-1}$ of this capacitance when the current density was increased to $10 \mathrm{~A} \mathrm{~g}^{-1}$ (Fig. S24c). As shown in Fig. S24d, the SSC device offers a maximum energy density of $16.6 \mathrm{~W} \mathrm{~h} \mathrm{~kg}^{-1}$ at a power density of $403 \mathrm{~W} \mathrm{~kg}^{-1}$.

\section{CONCLUSIONS}

In summary, we report the facile synthesis of azo-azaacene CMP via reductive nitro-coupling under hydrothermal conditions for
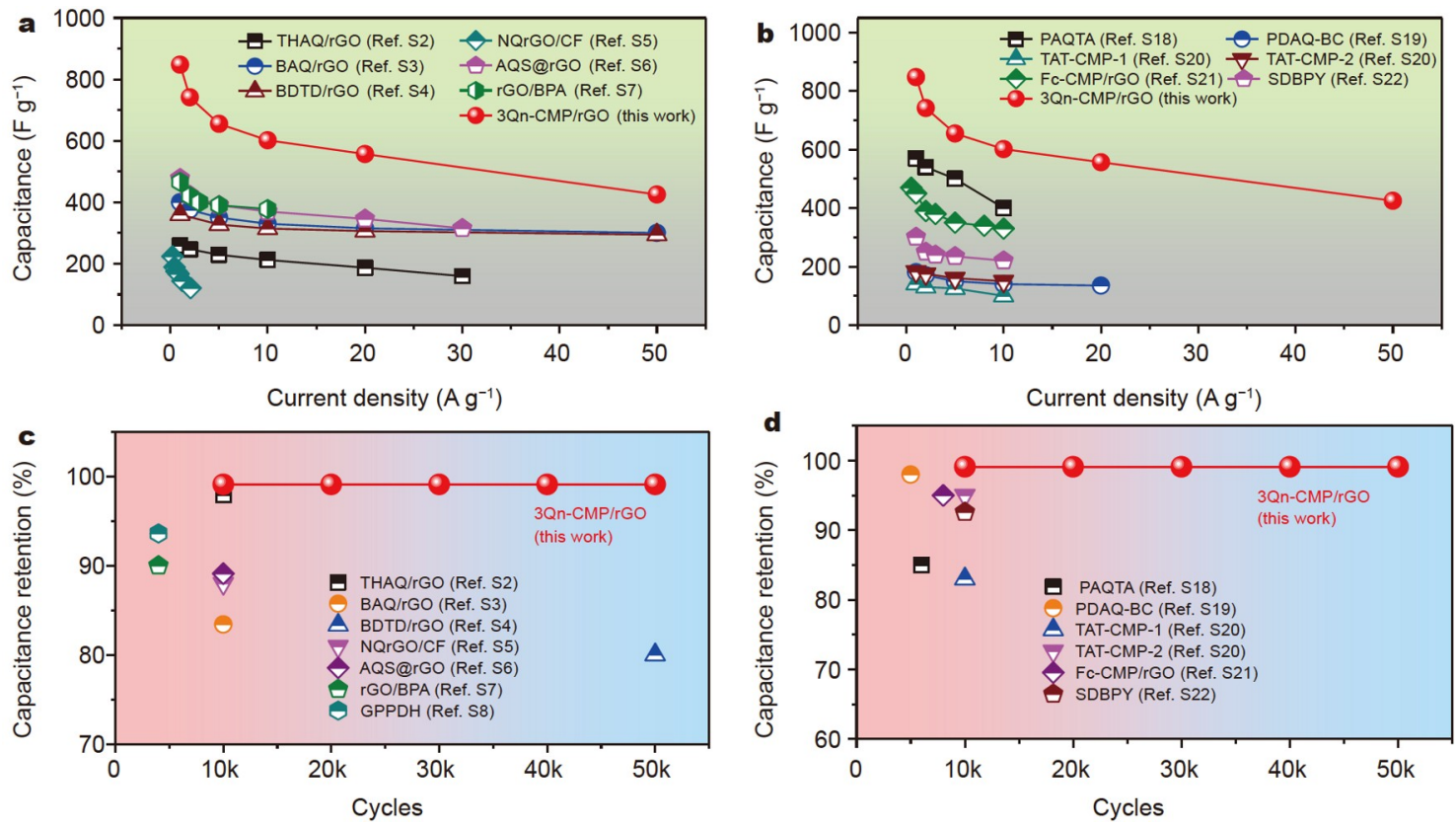

Figure 5 Comparison of the capacitance and rate capability ( $a, b)$ and cycling stability (c, d) of the synthesized CMP/rGO with representative redox molecule/rGO- or CMP-based electrodes published in the literature (the reference numbers refer to the Supplementary information). 
applications as electrochemical energy storage systems. The obtained 3Qn-CMP presented as 2D nanosheets with a thickness of $3.7-5.4 \mathrm{~nm}$ and a central core diameter of $\sim 1.6 \mathrm{~nm}$. The intercalation-initiated self-assembly and in-situ growth of redox CMP onto rGO nanosheets afforded 3Qn-CMP/rGO hybrids with elaborate hierarchical structures featuring CMP microspheres anchored on graphene layers. The azo linkage-enriched ion reservoir, together with the redox imine groups in large fused-azaacene cores, endowed CMP with dual active sites for electrochemical reactions. Coupling with the rGO substrate remarkably increased the porosity and charge conductivity of the resultant products, thereby dramatically improving their electrochemical dynamics and stability. 3Qn-CMP/rGO achieved a maximum $C_{s}$ of $847.8 \mathrm{~F} \mathrm{~A}^{-1}$ at $1 \mathrm{~A} \mathrm{~g}^{-1}$ and retained over $99 \%$ of its capacitance at $50 \mathrm{~A} \mathrm{~g}^{-1}$ after 50,000 charge/discharge cycles. To the best of our knowledge, our hybrid outperforms most other CMPs or redox organic electrodes reported in the literature in terms of $C_{s}$ and cycling stability. We believe that our redox azo-CMP strategy could readily be adopted for the design of new azo-CMPs with tailor-made architectures. Careful structure-modulation of redox skeletons and channels could produce superior CMP electrodes for electrochemical energy storage.

\section{Received 28 July 2021; accepted 15 October 2021; published online 26 November 2021}

1 Lee S, Hong J, Kang K. Redox-active organic compounds for future sustainable energy storage system. Adv Energy Mater, 2020, 10: 2001445

2 Zhao-Karger Z, Gao P, Ebert T, et al. New organic electrode materials for ultrafast electrochemical energy storage. Adv Mater, 2019, 31: 1806599

3 Huang J, Dong X, Guo Z, et al. Progress of organic electrodes in aqueous electrolyte for energy storage and conversion. Angew Chem Int Ed, 2020, 59: 18322-18333

4 Zhong L, Fang Z, Shu C, et al. Redox donor-acceptor conjugated microporous polymers as ultralong-lived organic anodes for rechargeable air batteries. Angew Chem Int Ed, 2021, 60: 10164-10171

5 Dai Y, Li W, Chen Z, et al. An air-stable electrochromic conjugated microporous polymer as an emerging electrode material for hybrid energy storage systems. J Mater Chem A, 2019, 7: 16397-16405

$6 \mathrm{Wu} \mathrm{X}$, Huang B, Wang Q, et al. Thermally chargeable supercapacitor using a conjugated conducting polymer: Insight into the mechanism of charge-discharge cycle. Chem Eng J, 2019, 373: 493-500

7 Zhao C, Chen Z, Wang W, et al. In situ electropolymerization enables ultrafast long cycle life and high-voltage organic cathodes for lithium batteries. Angew Chem Int Ed, 2020, 59: 11992-11998

8 Kou Y, Xu Y, Guo Z, et al. Supercapacitive energy storage and electric power supply using an aza-fused $\pi$-conjugated microporous framework. Angew Chem, 2011, 123: 8912-8916

9 Khattak AM, Sin H, Ghazi ZA, et al. Controllable fabrication of redoxactive conjugated microporous polymers on reduced graphene oxide for high performance faradaic energy storage. J Mater Chem A, 2018, 6: 18827-18832

10 Wang HG, Li Q, Wu Q, et al. Conjugated microporous polymers with bipolar and double redox-active centers for high-performance dual-ion, organic symmetric battery. Adv Energy Mater, 2021, 11: 2100381

11 Liao $\mathrm{Y}$, Wang $\mathrm{H}$, Zhu $\mathrm{M}$, et al. Efficient supercapacitor energy storage using conjugated microporous polymer networks synthesized from Buchwald-Hartwig coupling. Adv Mater, 2018, 30: 1705710

12 Lyu W, Zhang W, Liu $\mathrm{H}$, et al. Conjugated microporous polymer network grafted carbon nanotube fibers with tunable redox activity for efficient flexible wearable energy storage. Chem Mater, 2020, 32: 82768285

13 Lee JSM, Cooper AI. Advances in conjugated microporous polymers.
Chem Rev, 2020, 120: 2171-2214

14 Li K, Li Q, Wang Y, et al. An aromatic carbonyl compound-linked conjugated microporous polymer as an advanced cathode material for lithium-organic batteries. Mater Chem Front, 2020, 4: 2697-2703

15 Luo LW, Zhang C, Xiong P, et al. A redox-active conjugated microporous polymer cathode for high-performance lithium/potassium-organic batteries. Sci China Chem, 2021, 64: 72-81

16 Ouyang Z, Tranca D, Zhao Y, et al. Quinone-enriched conjugated microporous polymer as an organic cathode for Li-ion batteries. ACS Appl Mater Interfaces, 2021, 13: 9064-9073

17 Huangfu C, Liu Z, Lu X, et al. Strong oxidation induced quinone-rich dopamine polymerization onto porous carbons as ultrahigh-capacity organic cathode for sodium-ion batteries. Energy Storage Mater, 2021, 43: $120-129$

18 Molina A, Patil N, Ventosa E, et al. New anthraquinone-based conjugated microporous polymer cathode with ultrahigh specific surface area for high-performance lithium-ion batteries. Adv Funct Mater, 2020, 30: 1908074

19 Ajjan FN, Khan Z, Riera-Galindo S, et al. Doped conjugated polymer enclosing a redox polymer: Wiring polyquinones with poly(3,4-ethylenedioxythiophene). Adv Energy Sustain Res, 2020, 1: 2000027

20 Vitaku E, Gannett CN, Carpenter KL, et al. Phenazine-based covalent organic framework cathode materials with high energy and power densities. J Am Chem Soc, 2020, 142: 16-20

21 Wang W, Kale VS, Cao Z, et al. Phenanthroline covalent organic framework electrodes for high-performance zinc-ion supercapattery. ACS Energy Lett, 2020, 5: 2256-2264

$22 \mathrm{Ma} \mathrm{W}$, Luo LW, Dong $\mathrm{P}$, et al. Toward high-performance dihydrophenazine-based conjugated microporous polymer cathodes for dual-ion batteries through donor-acceptor structural design. Adv Funct Mater, 2021, 31: 2105027

23 Luo C, Xu GL, Ji X, et al. Reversible redox chemistry of azo compounds for sodium-ion batteries. Angew Chem Int Ed, 2018, 57: 2879-2883

24 Luo C, Ji X, Hou S, et al. Azo compounds derived from electrochemical reduction of nitro compounds for high performance Li-ion batteries. Adv Mater, 2018, 30: 1706498

25 Yang W, Ling B, Hu B, et al. Synergistic N-heterocyclic carbene/palladium-catalyzed umpolung 1,4-addition of aryl iodides to enals. Angew Chem Int Ed, 2020, 59: 2

26 Zhang L, Qian Y, Feng R, et al. Reversible redox chemistry in azobenzene-based organic molecules for high-capacity and long-life nonaqueous redox flow batteries. Nat Commun, 2020, 11: 3843

27 Huang S, Hu Y, Tan LL, et al. Highly C2/C1-selective covalent organic frameworks substituted with azo groups. ACS Appl Mater Interfaces, 2020, 12: 51517-51522

28 Chandra S, Kundu T, Kandambeth S, et al. Phosphoric acid loaded azo $(-\mathrm{N}=\mathrm{N}-)$ based covalent organic framework for proton conduction. J Am Chem Soc, 2014, 136: 6570-6573

29 Das G, Prakasam T, Addicoat MA, et al. Azobenzene-equipped covalent organic framework: Light-operated reservoir. J Am Chem Soc, 2019, 141: 19078-19087

30 Zhao G, Zhang Y, Gao Z, et al. Dual active site of the azo and carbonylmodified covalent organic framework for high-performance Li storage. ACS Energy Lett, 2020, 5: 1022-1031

31 Lu J, Zhang J. Facile synthesis of azo-linked porous organic frameworks via reductive homocoupling for selective $\mathrm{CO}_{2}$ capture. J Mater Chem $\mathrm{A}$, 2014, 2: 13831-13834

32 Arab P, Rabbani MG, Sekizkardes AK, et al. Copper(I)-catalyzed synthesis of nanoporous azo-linked polymers: Impact of textural properties on gas storage and selective carbon dioxide capture. Chem Mater, 2014, 26: 1385-1392

33 Liu $\mathrm{M}$, Yao C, Liu C, et al. $\mathrm{Ag}^{+}$doped into azo-linked conjugated microporous polymer for volatile iodine capture and detection of heavy metal ions. Sci Rep, 2018, 8: 14072

34 Peng C, Ning GH, Su J, et al. Reversible multi-electron redox chemistry of $\pi$-conjugated $\mathrm{N}$-containing heteroaromatic molecule-based organic cathodes. Nat Energy, 2017, 2: 17074

35 Bosch-Navarro C, Coronado E, Martí-Gastaldo C, et al. Influence of the $\mathrm{pH}$ on the synthesis of reduced graphene oxide under hydrothermal 
conditions. Nanoscale, 2012, 4: 3977-3982

36

Li C, Yang J, Pachfule P, et al. Ultralight covalent organic framework/ graphene aerogels with hierarchical porosity. Nat Commun, 2020, 11: 4712

37 Wu X, Han X, Liu Y, et al. Control interlayer stacking and chemical stability of two-dimensional covalent organic frameworks via steric tuning. J Am Chem Soc, 2018, 140: 16124-16133

38 Karnan M, Balachandran V, Murugan M. FT-IR, Raman and DFT study of 5-chloro-4-nitro-o-toluidine and $\mathrm{NBO}$ analysis with other halogen (Br, F) substitution. J Mol Structure, 2013, 1039: 197-206

39 Li X, Zhang C, Cai S, et al. Facile transformation of imine covalent organic frameworks into ultrastable crystalline porous aromatic frameworks. Nat Commun, 2018, 9: 2998

40 Batich CD, Donald DS. X-ray photoelectron spectroscopy of nitroso compounds: Relative ionicity of the closed and open forms. J Am Chem Soc, 1984, 106: 2758-2761

41 Urzúa J, Carbajo J, Yáñez C, et al. Electrochemistry and XPS of 2,7dinitro-9-fluorenone immobilized on multi-walled carbon nanotubes. J Solid State Electrochem, 2016, 20: 1131-1137

42 Corma A, Serna P. Chemoselective hydrogenation of nitro compounds with supported gold catalysts. Science, 2006, 313: 332-334

43 Blaser HU. A golden boost to an old reaction. Science, 2006, 313: $312-$ 313

44 Kim S, Choi HC. Light-promoted synthesis of highly-conjugated crystalline covalent organic framework. Commun Chem, 2019, 2: 60

45 Liu Y, Zhou J, Tang J, et al. Three-dimensional, chemically bonded polypyrrole/bacterial cellulose/graphene composites for high-performance supercapacitors. Chem Mater, 2015, 27: 7034-7041

46 Liu Y, Zhou J, Zhu E, et al. Covalently intercalated graphene oxide for oil-water separation. Curr Alzheimer Resbon, 2015, 82: 264-272

47 Mathis TS, Kurra N, Wang X, et al. Energy storage data reporting in perspective-Guidelines for interpreting the performance of electrochemical energy storage systems. Adv Energy Mater, 2019, 9: 1902007

48 Isikli S, Díaz R. Substrate-dependent performance of supercapacitors based on an organic redox couple impregnated on carbon. J Power Sources, 2012, 206: 53-58

49 Song Y, Liu T, Li M, et al. Engineering of mesoscale pores in balancing mass loading and rate capability of hematite films for electrochemical capacitors. Adv Energy Mater, 2018, 8: 1801784

50 Yan L, Zhao C, Sha Y, et al. Electrochemical redox behavior of organic quinone compounds in aqueous metal ion electrolytes. Nano Energy, 2020, 73: 104766

51 Sun B, Liu J, Cao A, et al. Interfacial synthesis of ordered and stable covalent organic frameworks on amino-functionalized carbon nanotubes with enhanced electrochemical performance. Chem Commun, 2017, 53: 6303-6306

52 Lei $\mathrm{Z}$, Yang Q, Xu Y, et al. Boosting lithium storage in covalent organic framework via activation of 14-electron redox chemistry. Nat Commun, 2018, 9: 576

53 Xu F, Jin S, Zhong H, et al. Electrochemically active, crystalline, mesoporous covalent organic frameworks on carbon nanotubes for synergistic lithium-ion battery energy storage. Sci Rep, 2015, 5: 8225

54 Shi R, Han C, Duan H, et al. Redox-active organic sodium anthraquinone-2-sulfonate (AQS) anchored on reduced graphene oxide for high-performance supercapacitors. Adv Energy Mater, 2018, 8: 1802088

$55 \mathrm{Lu} \mathrm{X}$, Li L, Song B, et al. Mechanistic investigation of the graphene functionalization using $p$-phenylenediamine and its application for supercapacitors. Nano Energy, 2015, 17: 160-170

56 Chandra S, Roy Chowdhury D, Addicoat M, et al. Molecular level control of the capacitance of two-dimensional covalent organic frameworks: Role of hydrogen bonding in energy storage materials. Chem Mater, 2017, 29: 2074-2080

Acknowledgements This work was supported by the National Natural Science Foundation of China (51861145401 and 21905136) and the Priority Academic Program Development (PAPD) of Jiangsu Higher Education Institutions.
Author contributions Tang W and Zhou J led the whole project. Zhou J conceived the concept of material design. Fang D synthesized and tested the samples. Zhang $\mathrm{S}$ contributed to the sample preparation. Fang D proceeded the writing of the manuscript with contributions from Wang X, Sheng L and Tang J. All authors contributed to the general discussion.

Conflict of interest The authors declare that they have no conflict of interest.

Supplementary information Experimental details and supporting data are available in the online version of this paper.

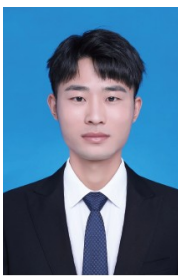

Dianlei Fang received his BS degree in 2021 from Nanjing University of Science and Technology. His research interests include the molecular engineering of redox organic electrodes and their applications in electrochemical energy storage.

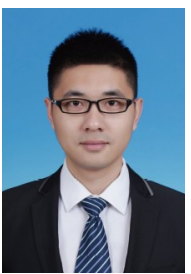

Jie Zhou received his $\mathrm{PhD}$ degree in chemical engineering and technology from Nanjing University of Science and Technology in 2016. He currently works as an associate professor at the university. His research interests mainly focus on the molecular design of organic semiconductors for energy conversion and storage.

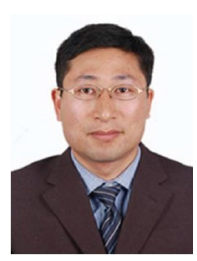

Weihua Tang has been a professor at Nanjing University of Science and Technology since 2009. He received his $\mathrm{PhD}$ degree in chemistry from the National University of Singapore. He was then trained as an independent researcher at the Institute of Materials Research and Engineering in Singapore and the University of Melbourne. His research interests mainly focus on the molecular design of conjugated materials and devices for organic/perovskite solar cells and energy storage systems.

\section{无催化剂硝基偶联合成偶氮基共轭微孔聚合物及其 水系储能研究}

房佃磊, 王金歪, 张士杰, 盛玲艳, 唐键, 周杰*, 唐卫华*

摘要 发展可构建材料结构的方法对于共轭微孔聚合物(CMP)具有重 要意义. 目前, 以偶氮键桥联氮杂并苯共轭微孔聚合物的合成仍具有极 大的挑战. 在此, 我们以硝基化二喹喔啉并 $\left[2,3-\mathrm{a}: 2^{\prime}, 3^{\prime}-\mathrm{c}\right]$ 吩嗪为单体, 在 $N, N$-二甲基甲酰胺与水混合溶剂中 $(1: 3, v / v)$ 一步水热还原合成偶氮基 共价有机框架 (孔径为 $1.6 \mathrm{~nm}$ )。通过原位一步还原氧化石墨烯 $(\mathrm{rGO})$ 进行插层与自组装, 获得的 $3 \mathrm{Qn}-\mathrm{CMP} / \mathrm{rGO}$ 复合材料中 CMP微球 $($ 粒径 0.5-1.5 $\mu \mathrm{m}$ ) 针定于 $\mathrm{rGO}$ 纳米片层上 ( 3 层). 该多孔复合物具有偶氮和亚 胺双重氧化还原位点及高电荷传导能力. $3 \mathrm{Qn}-\mathrm{CMP}$ 和 $3 \mathrm{Qn}-\mathrm{CMP} / \mathrm{rGO}$ 均在酸性水系电解质中表现出优异的电荷储存能力, 倍率性 $\left(1-50 \mathrm{~A} \mathrm{~g}^{-1}\right)$ 和长期循环稳定性. 在 $1 \mathrm{~A} \mathrm{~g}^{-1}$ 的电流密度下, 它们分别实现 615.4 和 $847.8 \mathrm{~F} \mathrm{~g}^{-1}$ 的比电容, 在 $50 \mathrm{~A} \mathrm{~g}^{-1}$ 下充放电 50,000 次后的保留率高达 $99.1 \%$. 据 我们所知, 该比容量和循环稳定性优于文献中多数有机分子或CMP复合材 料. 该工作提供了多重结构偶氮共轭多孔聚合物材料及复合物的普适性合 成策略, 有望应用于电化学储能和催化等领域. 